\title{
Prevalence of Extended-Spectrum and Metallo $\beta$-Lactamase Production in AmpC $\beta$-Lactamase Producing Pseudomonas aeruginosa Isolates From Burns
}

\author{
Roya Rafiee ${ }^{1}$; Fereshteh Eftekhar ${ }^{1,}$; Seyyed Ahmad Tabatabaei ${ }^{2}$; Dariush Minaee Tehrani ${ }^{1}$ \\ ${ }^{1}$ Department of Microbiology, Faculty of Biological Sciences, Shahid Beheshti University, Tehran, IR Iran \\ ${ }^{2}$ Department of Pediatrics, Shahid Beheshti University of Medical Sciences, Tehran, IR Iran \\ ${ }^{*}$ Corresponding author: Fereshteh Eftekhar, Department of Microbiology, Faculty of Biological Sciences, Shahid Beheshti University, Tehran, IR Iran. Tel: +98-2129903208, Fax: +98- \\ 2122431664, E-mail: f-eftekhar@sbu.ac.ir
}

Received: December 15, 2013; Revised: June 09, 2014; Accepted: June 21, 2014

\begin{abstract}
Background: Pseudomonas aeruginosa is one of the most common causes of nosocomial infections. Resistance of P. aeruginosa to $\beta$-lactam antibiotics may be the result of acquired resistance through mutation and over production of various antibiotic inactivating enzymes. This research aimed to determine the prevalence of extended-spectrum $\beta$-lactamases (ESBL) and metallo $\beta$-lactamase (MBL) production as well as the presence of their related genes among AmpC $\beta$-lactamase producing P. aeruginosa isolated from burns.

Objectives: The current study aimed to determine the prevalence of class A ESBL and MBL production in relation to the presence of their related genes among AmpC $\beta$-lactamase producing P. aeruginosa isolated from burns.

Materials and Methods: The antimicrobial susceptibility of 51 P. aeruginosa isolates from patients with burns was examined against 13 antibiotics by the disc diffusion method. Minimum inhibitory concentrations (MIC) for imipenem and ceftazidime were measured by the microdilution method. AmpC production was detected by AmpC disc and the modified three-dimensional extract tests. ESBL phenotype was confirmed by the double disc synergy test (DDST). Presence of $\beta$-lactamase genes was detected by specific primers and polymerase chain reaction (PCR).

Results: All isolates were multidrug resistant. AmpC, ESBL and MBL production were observed in 35 (68.6\%), 20 (39.2\%) and 19 (37.3\%) isolates, respectively. Overall, 43 isolates (84.3\%) carried $\beta$-lactamase genes, out of which 31 (60.8\%) harbored bla $A m p C, 20(39.2 \%)$ had bla ${ }_{T E M}$ and $11(21.6 \%)$ carried bla ${ }_{P E R^{-1}}$ genes. Among the AmpC producers, two isolates (6.5\%) carried bla ${ }_{A m p C}+$ bla $_{E S B L}{ }^{13}(41.9 \%)$ had bla $_{A m p C}{ }^{+}$ bla $_{M B L}$ and six (19.4\%) produced the three enzymes.

Conclusions: A high prevalence of multiple $\beta$-lactamase production was observed among the AmpC producers (60\%), of which the majority co-produced AmpC and MBL. The current study results showed correlation between $\beta$-lactamase production and the presence of antibiotic resistance genes in the isolates.
\end{abstract}

Keywords:Pseudomonas aeruginosa; Beta-Lactamase; blaAMPC; blaESBL; blaMBL

\section{Background}

Pseudomonas aeruginosa is an important opportunistic pathogen with the ability to propagate on medical devices, hospital environments and even in disinfectants (1). Due to the intrinsic resistance to many antimicrobial agents, $P$. aeruginosa infections present serious therapeutic challenges both in the community and health centers. In addition, the organism can acquire resistance elements to multiple classes of antibacterial agents, even during the course of treatment $(2,3)$. P. aeruginosa isolates from burn wounds are often responsible for systemic sepsis, graft loss, prolonged hospital stay and mortality (4).

Several mechanisms are responsible for resistance to $\beta$-lactam antibiotics in $P$. aeruginosa including: genetic mutations that lead to stable over expression of chromosome-mediated AmpC cephalosporinases, acquisition of transferable $\beta$-lactamase genes, overproduction of efflux systems, and reduced permeability (5). Among the numerous $\beta$-lactamases, Ambler class A extended-spectrum $\beta$-lactamases (ESBLs) and class B metallo $\beta$-lactamases (MBLs) are reported as rapidly growing enzymes in clinical isolates of $P$. aeruginosa. ESBLs are capable of hydrolyzing penicillins, cephalosporins, and aztreonam (except for cephamycins or carbapenems). These enzymes are inhibited by $\beta$-lactamase inhibitors such as clavulanic acid $(6,7)$. Among more than 200 different known ESBLs in Gram-negative bacteria, 32 are detected in $P$. aeruginosa belonging to class A (TEM, SHV, CTX-M, PER, VEB, GES, BEL), and class D (OXA type) $\beta$-lactamases (8).

AmpC $\beta$-lactamases hydrolyze cephamycins (e.g. cefoxitin and cefotetan), oxyimino-cephalosporins (e.g. ceftazidime, cefotaxime, and ceftriaxone), and monobactams (e.g. aztreonam) (9). In P. aeruginosa, decreased susceptibility to the extended-spectrum cephalosporins such as ceftazidime mostly results from over expression of the naturally occurring AmpC $\beta$-lactamases (10). Among the carbapenems, intermediate susceptibility to imipenem is widely associated with the modification of the outer

Copyright (C 2014,Ahvaz Jundishapur University of Medical Sciences; Published by Kowsar Corp. This is an open-access article distributed under the terms of the Creative Commons Attribution License, which permits unrestricted use, distribution, and reproduction in any medium, provided the original work is properly cited. 
membrane protein OprD, production of MBLs and over expression of the cephalosporinases associated with decreased outer membrane permeability. Recently, a novel resistance mechanism was shown in $P$. aeruginosa, where peculiar AmpC $\beta$-lactamases with expanded-spectrum activity toward imipenem may also contribute to decreased imipenem susceptibility (11).

\section{Objectives}

The current study aimed to determine the prevalence of ESBL and MBL production among AmpC $\beta$-lactamase producing $P$. aeruginosa isolated from burns and evaluate the presence of their related antibiotic resistance genes.

\section{Materials and Methods}

\subsection{Bacterial Isolates}

Fifty one non-duplicate $P$. aeruginosa isolates were randomly selected from a collection of burn isolates provided by Shahid Motahari Hospital in 2011. The antibiotic susceptibility patterns and MBL production were previously determined using disc diffusion and the double disc synergy tests, respectively (12). The isolates were maintained in brain heart infusion broth (Oxoid, UK) containing $10 \%$ dimethyl sulfoxide and stored at $-20^{\circ} \mathrm{C}$ until use. Controls for polymerase chain reaction (PCR) tests were: $P$. aeruginosa $\mathrm{PAO}$ containing bla $_{\mathrm{AmpC}}$ (provided by Dr. Abdi, Al-Zahra University, Iran), DNA from Klebsiella pneumoniae 7881 harboring bla ${ }_{T E M}$, bla $_{S H V}$ and bla ${ }_{C T X-M}$, $P$. aeruginosa KOAS carrying blaPER-1 and P. aeruginosa 10.2 harboring bla $V E B-1$ were obtained from Pasteur Institute, Tehran, Iran. P. aeruginosa ATCC 27853 was also used as the susceptible control strain.

\subsection{Antimicrobial Susceptibility Testing}

Antibiotic susceptibility testing of the isolates was carried out by the disc diffusion method, according to the Clinical and Laboratory Standards Institute guidelines (CLSI) (5). The used antibiotic discs (MAST Group LTD, Merseyside, UK) were: ceftazidime $(30 \mu \mathrm{g})$, aztreonam (30 $\mu \mathrm{g})$, carbenicillin $(100 \mu \mathrm{g})$, piperacillin $(100 \mu \mathrm{g})$, ticarcillin $(75 \mu \mathrm{g})$, cotrimoxazole $(25 \mu \mathrm{g})$, amikacin $(30 \mu \mathrm{g})$, cefepime $(30 \mu \mathrm{g})$, ciprofloxacin $(5 \mu \mathrm{g})$, tobramycin $(10 \mu \mathrm{g})$, meropenem $(10 \mu \mathrm{g})$, imipenem $(10 \mu \mathrm{g})$, cefoxitin $(30 \mu \mathrm{g})$, and piperacillin/tazobactam $(100 / 10 \mu \mathrm{g})$.

\subsection{Determination of Minimum Inhibitory Concen- trations}

Minimum inhibitory concentrations (MICs) for imipenem and ceftazidime were determined by the microdilution assay as recommended by the CLSI (13).

\subsection{Phenotypic Detection of AmpC Production}

Initial screening for AmpC $\beta$-lactamase production was performed by the AmpC disc test. The results showed a zone diameter of $<18 \mathrm{~mm}$ for cefoxitin (14). Briefly, a blank disc moistened with sterile saline, was inoculated with a few colonies of the test strain. The disc was then placed next to a cefoxitin disc $(30 \mu \mathrm{g})$ on the surface of a Muller Hinton agar plate (Leofilchem, Italy) previously inoculated with a lawn of Escherichia coli ATCC 25922. The plate was incubated overnight at $37^{\circ} \mathrm{C}$. An indentation of the cefoxitin inhibition zone adjacent to the disc containing the test strain was an indication of AmpC $\beta$-lactamase production.

AmpC production was also determined using a modified three-dimensional extract test (TDET) (15). Briefly, bacterial colonies from overnight cultures on Mueller Hinton agar were transferred to a pre-weighed sterile micro centrifuge tube. The tube was weighed again to obtain 10 to $15 \mathrm{mg}$ of bacterial wet weight per sample. The bacteria were then suspended in peptone water (Merck, Germany) and centrifuged at $3000 \mathrm{rpm}$ (Sigma 1-13 microcentrifuge, Germany) for 15 minutes. Crude cell extracts were prepared by 10 cycles of alternate freeze-thawing at $-78^{\circ} \mathrm{C}$ (dry ice/ethanol bath) and $37^{\circ} \mathrm{C}$. The extracts were then used to screen for AmpC using the following method. Linear slits $(3 \mathrm{~cm})$ were cut into plates inoculated with each test organism using a sterile scalpel blade, $3 \mathrm{~mm}$ away from a cefoxitin disc in an outward radial direction. Approximately 40 to $50 \mu \mathrm{L}$ of the prepared extract was loaded into each slit and the plates were kept upright for 5 to 10 minutes followed by incubation at $37^{\circ} \mathrm{C}$ overnight. AmpC production was recorded when clear distortion of cefoxitin inhibition zones were observed (Figure 1).

\subsection{Phenotypic Detection of ESBL}

ESBL production was detected by the conventional double disc synergy test (DDST) using ceftazidime $(30 \mu \mathrm{g})$ and cefotaxime discs $(30 \mu \mathrm{g})$ with or without clavulanic acid $(10 \mu \mathrm{g})$ as recommended by the CLSI. An increase of $\geq 5$ $\mathrm{mm}$ in the inhibition zones of either cephalosporin in combination with clavulanic acid compared to the cephalosporin alone was interpreted as ESBL production (13).

\subsection{PCR Amplification of AmpC and ESBL Genes}

DNA extraction was carried out by boiling. Briefly, a loopful of an overnight grown culture of each test isolate as well as the control strains were suspended in $500 \mu \mathrm{L}$ of sterile double distilled water, boiled at $100^{\circ} \mathrm{C}$ for $10 \mathrm{~min}$ utes and were centrifuged at $12^{\prime} 000 \times \mathrm{g}$ for 10 minutes. The supernatants were then used as DNA template for PCR amplification tests.

PCR amplifications were performed using specific primers for Ambler class A (bla TEM , bla ${ }_{S H V}$, bla ${ }_{C T X^{-M}}$, bla ${ }_{V E B-1}$, bla $_{P E R-1}$ ) and class $C\left(\right.$ bla $\left._{A m p C}\right) \beta$-lactamase genes (Table 1). AmpC amplifications were initially performed using a pair of external primers (PreAmpC-PA1 and PostAmpCPA2) yielding a 1243-base pair product encompassing the entire AmpC gene of $P$. aeruginosa, but excluding its 
Rafiee Ret al.

promoter sequences. Another pair of primers (AmpC-PA$A$ and AmpC-PA-B) was also used as internal primers for AmpC gene yielding a 1063 bp product (Table 1).

PCR experiments were carried out in $25 \mu \mathrm{L}$ volume reaction mixtures containing $10 \mathrm{pM}$ of each primer, $200 \mu \mathrm{M}$ dNTP, $1.4 \mathrm{mM} \mathrm{MgCl}_{2}, 1 \mu \mathrm{L}$ of crude DNA template and $1 \mathrm{U}$ of Taq DNA polymerase in the reaction buffer provided by the manufacturer (CinnaGen, Tehran, Iran). Amplifications were carried out in thermocycler (Techne, UK) using the following program: five minutes denaturation at $95^{\circ} \mathrm{C}$ followed by 30 cycles of $95^{\circ} \mathrm{C}$ for one minute, one minute at the annealing temperature $\left(43\right.$ to $63^{\circ} \mathrm{C}$ for ES-
BLs, $62^{\circ} \mathrm{C}$ and/or $65^{\circ} \mathrm{C}$ for AmpC), and one minute at $72^{\circ} \mathrm{C}$ with a final extension period of 10 minutes at $72^{\circ} \mathrm{C}$.

\subsection{Statistical Analyses}

Comparisons of $\beta$-lactamase phenotypes and presence of the related genes were performed using the chi-square test. Correlation between antibiotic resistance and $\beta$-lactamase gene carriage was analyzed by the nonparametric Mann-Whitney U test. All statistical analyses were carried out using SPSS software version 19. A P Value of $<$ 0.05 was considered statistically significant.

\begin{tabular}{|c|c|c|c|}
\hline$\beta$-lactamase (Primer) & Sequence (5' to $3^{\prime}$ ) & Product Size (bp) & Reference \\
\hline \multicolumn{4}{|l|}{ Class A } \\
\hline TEM-F & GAGTATTCAACATTTCCGTGTC & 851 & (10) \\
\hline TEM-R & TAATCAGTGAGGCACCTATCTC & & \\
\hline SHV-F & AAGATCCACTATCGCCAGCAG & 231 & (10) \\
\hline SHV-R & ATTCAGTTCCGTTTCCCAGCGG & & \\
\hline CTX-M-F & CGCTTTGCGATGTGCAG & 550 & (16) \\
\hline CTX-M-R & ACCGCGATATCGTTGGT & & \\
\hline PER-1-F & ATGAATGTCATTATAAAAGC & 920 & (10) \\
\hline PER-1-R & AATTTGGGCTTAGGGCAGAA & & \\
\hline VEB-1-F & CGACTTCCATTTCCCGATGC & 643 & (10) \\
\hline VEB-1-R & GGACTCTGCAACAAATACGC & & \\
\hline \multicolumn{4}{|l|}{ Class C } \\
\hline PreAmpC-PA1 & ATGCAGCCAACGACAAAGG & 1243 & (11) \\
\hline PostAmpC-PA2 & CGCCCTCGCGAGCGCGCTTC & & \\
\hline ampC-PA-A & CTTCCACACTGCTGTTCGCC & 1063 & (11) \\
\hline ampC-PA-B & TTGGCCAGGATCACCAGTCC & & \\
\hline
\end{tabular}

Figure 1. Modified Three-Dimensional Test for Phenotypic Detection of AmpC $\beta$-Lactamase

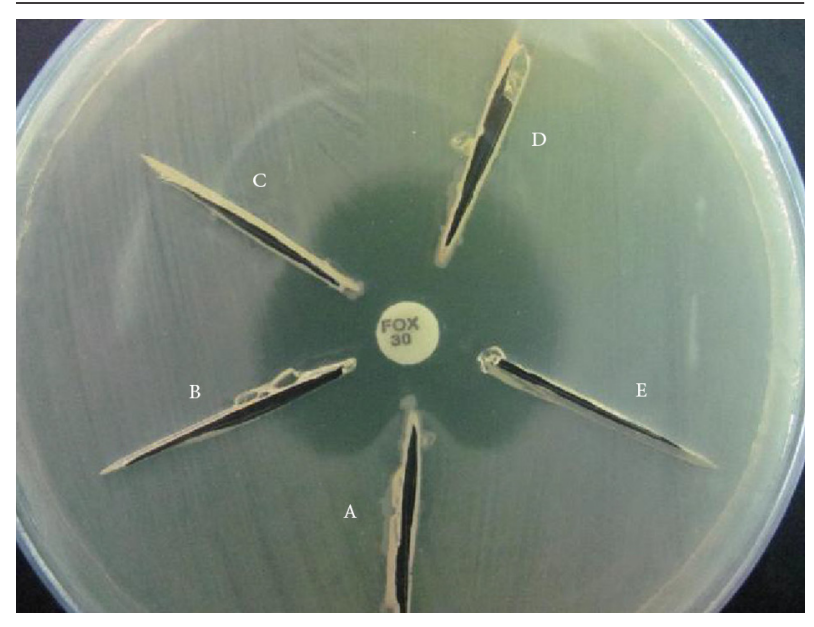

A; clear distortion of inhibition zone indicates AmpC $\beta$-lactamase production, B, C and D; absence of distortion indicates negative test. E; negative control

\section{Results}

Disc diffusion results showed that all test isolates were resistant to three or more antibiotic classes; therefore, they were identified as multidrug resistant (MDR). Antibiotic resistance rates shown in Figure 2 were: piperacilin, carbenicillin, tobramycin and cefoxitin (100\%), imipenem, ticarcillin, aztreonam and cotrimoxazole (98\%), amikacin, cefepime and ciprofloxacin (96\%), meropenem (94\%), ceftazidime (92\%) and piperacillin/tazobactam (88\%). The MIC results showed $88.2 \%$ resistance to ceftazidime and $98 \%$ to imipenem, confirming the results obtained by the disc diffusion method. There was no significant association between antibiotic resistance patterns and $\beta$-lactamase production. AmpC production was observed in 35 isolates (68.6\%) using the AmpC disc test and only 18 (51.4\%) by the TDET test suggesting the superiority of the AmpC disc test to TDET. ESBL production was observed in 20 isolates (39.2\%) and 19 isolates (37.3\%) were MBL producers. Of the 35 AmpC producers, two (5.7\%) had the ESBL phenotype, 13 (37.1\%) produced MBL and six (17.1\%) were positive for all three enzymes. 
PCR amplification results showed that 43 isolates (84.3\%) carried $\beta$-lactamase genes. Bla $A m p C$ was present in 31 isolates (60.8\%), two of which were susceptible to ceftazidime (Table 2). There was a significant correlation between the AmpC disc test phenotype and AmpC gene carriage ( $\mathrm{P}<0.05)$, while no relationship was observed between TDET and presence of the AmpC gene. Among the class A $\beta$-lactamases, 20 isolates (39.2\%) harbored blaTEM and 11 (21.6\%) carried bla PER-1 genes. SHV, CTX-M and VEB-1 genes were not detected in any of the isolates. All MBL producing isolates harbored their related genes (12). Overall, there was correlation between $\beta$-lactamase productions with the presence of their related genes in the test isolates $(\mathrm{P}<0.05)$.

Table 2. Distribution of $\beta$-Lactamase Genes Among Pseudomonas aeruginosa Burn Isolates

\begin{tabular}{|c|c|c|c|}
\hline$\beta$-Lactamases & $\begin{array}{c}\text { NO. (\%) } \\
\text { Isolates }\end{array}$ & Ceftazidime $^{a}$ & Imipenem $^{\mathrm{a}}$ \\
\hline AmpC alone & $10(19.6)$ & $\geq 256(1 / 10)$ & $16-64(0 / 10)$ \\
\hline$A m p C+$ ESBL $^{b}$ & $2(3.9)$ & $>256(0 / 2)$ & $64(0 / 2)$ \\
\hline $\mathbf{A m p C}+\mathbf{M B L}^{\mathrm{C}}$ & $13(25.5)$ & $>256(1 / 13)$ & $16-64(0 / 13)$ \\
\hline$A m p C+E S B L+M B L$ & $6(11.8)$ & 32 to $\geq 256(0 / 6)$ & $16-64(0 / 6)$ \\
\hline
\end{tabular}

Figure 2. Antibiotic Resistance Patterns of Pseudomonas aeruginosa Isolates from Burns

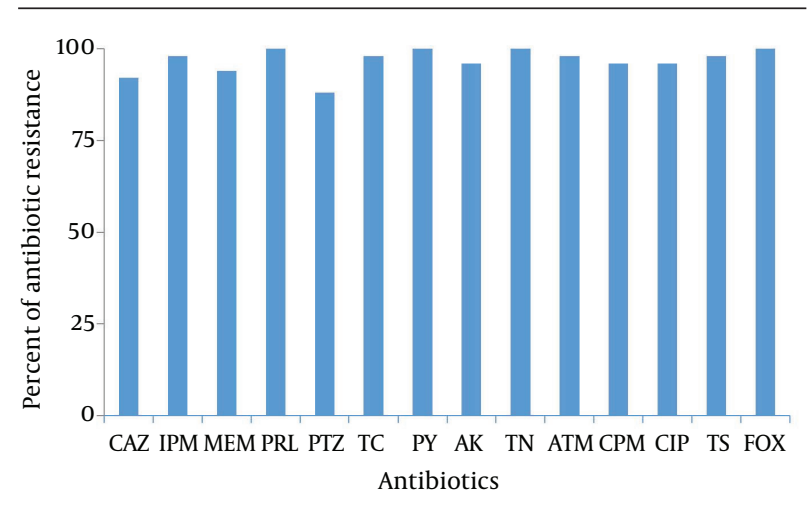

CAZ, ceftazidime; IPM, imipenem; MEM, meropenem; PRL, piperacilin; PTZ, piperacillin-tazobactam; TC, ticarcillin; PY, carbenicillin; AK, amikacin; TN, tobramycin; ATM, azteonam; CPM, cefepime; CIP, ciprofloxacin; TS, cotrimoxazole; FOX, cefoxitin.

\section{Discussion}

Multiple $\beta$-lactamase producing P. aeruginosa cause major therapeutic failure and pose significant clinical challenges if remain undetected (17). Among more than 800 $\beta$-lactamases identified in Gram-negative bacteria, at least 120 were detected in P. aeruginosa, among which, AmpC, ESBLs, and MBLs are clinically significant (8). The Current
CLSI guidelines do not describe a method for detection of AmpC $\beta$-lactamases. AmpC disc test was originally introduced to detect plasmid-mediated AmpC $\beta$-lactamases (18). However, Black et al. reported the detection of chromosomally mediated inducible AmpC $\beta$-lactamases in a number of bacteria including P. aeruginosa, by the AmpC disc test (19). In the present study, using the AmpC disc test, AmpC production (68.6\%) was higher among the $P$. aeruginosa isolates from burns compared to those of other reports (17.3-59.4\%) (17, 20-23). On the other hand, only half of the isolates which were positive in AmpC disc test showed AmpC production by TDET. Basak et al. also showed that the AmpC disc test was superior to TDET in screening for AmpC production (20).

The current study results showed a significant association between the AmpC phenotype determined by the AmpC disc test and AmpC gene carriage $(\mathrm{P}<0.05)$. The rate of AmpC gene carriage (60.8\%) in the present study was similar to a report from Taiwan, where presentation of AmpC gene in P. aeruginosa was 59.6\% (16). However, in a study conducted in Iran, Fazeli et al. reported 100\% AmpC gene carriage among 72 pediatric P. aeruginosa isolates from various clinical specimens, but did not show the AmpC phenotype (24). Gene presence does not necessarily mean its expression and depends on environmental conditions. Therefore, the current study believes that it is important to associate the enzyme phenotype with the presence of resistance genes.

Detection of ESBLs in AmpC producing Gram-negative bacteria is often a problem. High level expression of AmpC can prevent recognition of ESBLs leading to false negative results $(25,26)$. ESBL production in our isolates (39.2\%) was higher compared to the other reports from Iran (2.2-23.3\%) and other countries (1.81-17\%) (16, 27-31). Coproduction of AmpC and ESBL (3.9\%) in the isolates of this study was similar to the findings of Upadhyay et al. (3.3\%), but much lower than several other reports (24.5$26 \%)(17,22,23)$. Finally, similar to a report from Brazil, the isolates of the present study did not harbor SHV and CTX-M genes (32).

In the past 30 years, extensive use of carbapenems to treat AmpC and ESBL producing P. aeruginosa, has led to increased levels of bacterial resistance mostly due to production of MBLs $(9,33,34)$. Coproduction of AmpC and MBL was detected in $25.5 \%$ of the isolates which was low compared to the other studies worldwide (45.5-46.6\%) $(22,23)$. Alarmingly, $11.8 \%$ of the isolates of the current study produced AmpC along with ESBL and MBL. Detection of multiple $\beta$-lactamases in highly resistant bacteria could be useful for the selection of suitable antibiotic therapy and avoiding treatment failure as well as reducing mortality rates in hospitalized patients.

In conclusion, the present study showed a high prevalence of AmpC production among P. aeruginosa isolates from burn wounds (68.6\%). More importantly, multiple $\beta$-lactamase production was observed in $60 \%$ of the AmpC producers, the majority of which also produced 
Rafiee Ret al.

MBL (25.5\%). Co-production of AmpC with ESBL occurred in $3.9 \%$ of the isolates and $11.9 \%$ produced the three $\beta$-lactamases.

\section{Acknowledgements}

The authors wish to thank Shahid Beheshti University Research Council for providing a special grant to support this research, and also Shahid Motahari Hospital microbiology laboratory for providing the bacterial isolates.

\section{Authors' contributions}

The funder organization had no role in the design and conducting the study, collection, management and analysis of the data, preparation, review, and approval of the manuscript. Roya Rafiee (Ph.D student) carried out the research, collected the data and wrote a draft of the manuscript, Fereshteh Eftekhar (Ph.D supervisor) was responsible for designing the research project, interpretation of the results and final preparation of the manuscript, Seyyed Ahmad Tabatabaei (Ph.D adviser) provided some bacterial isolates and advised the choice of antibiotics, Dariush Minaee-Tehrani (Ph.D adviser) provided technical advice and support.

\section{Funding/Support}

This research was financially supported by Shahid Beheshti University Research Council.

\section{References}

1. Fluit AC, Verhoef J, Schmitz FJ. Antimicrobial resistance in European isolates of Pseudomonas aeruginosa. European SENTRY Participants. Eur J Clin Microbiol Infect Dis. 2000;19(5):370-4.

2. Lister PD, Wolter DJ, Hanson ND. Antibacterial-resistant Pseudomonas aeruginosa: clinical impact and complex regulation of chromosomally encoded resistance mechanisms. Clin Microbiol Rev. 2009;22(4):582-610.

3. Selim S, El Alfy S, Al Ruwaili M, Abdo A, Al Jaouni S. Susceptibility of imipenem-resistant Pseudomonas aeruginosa to flavonoid glycosides of date palm (Phoenix dactylifera L.) tamar growing in Al Madinah, Saudi Arabia. Afr J Biotechnol. 2012;11(2):416-22.

4. Coetzee E, Rode H, Kahn D. Pseudomonas aeruginosa burn wound infection in a dedicated paediatric burns unit. S Afr J Surg. 2013;51(2):50-3.

5. Livermore DM. Multiple mechanisms of antimicrobial resistance in Pseudomonas aeruginosa: our worst nightmare? Clin Infect Dis. 2002;34(5):634-40.

6. Bush K, Jacoby GA, Medeiros AA. A functional classification scheme for beta-lactamases and its correlation with molecular structure. Antimicrob Agents Chemother. 1995;39(6):1211-33.

7. Paterson DL, Bonomo RA. Extended-spectrum beta-lactamases: a clinical update. Clin Microbiol Rev. 2005;18(4):657-86.

8. Zhao WH, Hu ZQ. Beta-lactamases identified in clinical isolates of Pseudomonas aeruginosa. Crit Rev Microbiol. 2010;36(3):245-58.

9. Jacoby GA. AmpC beta-lactamases. Clin Microbiol Rev. 2009; 22(1):161-82.

10. Weldhagen GF, Poirel L, Nordmann P. Ambler class A extendedspectrum beta-lactamases in Pseudomonas aeruginosa: novel developments and clinical impact. Antimicrob Agents Chemother. 2003;47(8):2385-92.

11. Rodriguez-Martinez JM, Poirel L, Nordmann P. Extended-spectrum cephalosporinases in Pseudomonas aeruginosa. Antimicrob Agents Chemother. 2009;53(5):1766-71.

12. Salimi F, Eftekhar F. Coexistence of AmpC and extendedspectrum $\beta$-lactamases in metallo- $\beta$-lactamase producing Pseudomonas aeruginosa burn isolates in Tehran. Jundishapour J Microbiol. 2013;6(8).

13. Cockerill FR, Clinical.. Performance standards for antimicrobial susceptibility testing: twenty-second informational supplement;[... provides updated tables for... M02-A11 and M07-A9].: National Committee for Clinical Laboratory Standards; 2012.

14. Singhal S, Mathur T, Khan S, Upadhyay DJ, Chugh S, Gaind R, et al. Evaluation of methods for AmpC beta-lactamase in Gram negative clinical isolates from tertiary care hospitals. Indian JMed Microbiol. 2005;23(2):120-4.

15. Manchanda V, Singh NP. Occurrence and detection of AmpC beta-lactamases among Gram-negative clinical isolates using a modified three-dimensional test at Guru Tegh Bahadur Hospital, Delhi, India. J Antimicrob Chemother. 2003;51(2):415-8.

16. Lin SP, Liu MF, Lin CF, Shi ZY. Phenotypic detection and polymerase chain reaction screening of extended-spectrum betalactamases produced by Pseudomonas aeruginosa isolates. $J$ Microbiol Immunol Infect. 2012;45(3):200-7.

17. Upadhyay S, Sen MR, Bhattacharjee A. Presence of different betalactamase classes among clinical isolates of Pseudomonas aeruginosa expressing AmpC beta-lactamase enzyme. J Infect Dev Ctries. 2010;4(4):239-42.

18. Prevalence of plasmid-mediated AmpC $\beta$-lactamases in Klebsiella pneumoniae (KP), Klebsiella oxytoca (KO), Proteus mirabilis (PM), and Salmonella (S) isolates from 42 ICU and 21 non-ICU sites in the United States. In: Black JA, Moland ES, Hossain A, Lockhart TJ, Olson LB, Thamson. K. S. editors. Interscience Conference on Antimicrobial Agents and Chemotherapy (ICCAC). 2003.

19. Black JA, Moland ES, Thomson KS. AmpC disk test for detection of plasmid-mediated AmpC beta-lactamases in Enterobacteriaceae lacking chromosomal AmpC beta-lactamases. J Clin Microbiol. 2005; 43(7):3110-3.

20. Basak S, Khodke M, Bose S, Mallick SK. Inducible Amp C beta-lactamase producing Pseudomonas aeruginosa isolated in a rural hospital of central India. JClin Diagn Res. 2009;3:19-7.

21. Shahid M, Malik A, Agrawal M, Singhal S. Phenotypic detection of extended-spectrum and $\mathrm{AmpC}$ beta-lactamases by a new spot-inoculation method and modified three-dimensional extract test: comparison with the conventional three-dimensional extract test. J Antimicrob Chemother. 2004;54(3):684-7.

22. Kumar V, Sen MR, Nigam C, Gahlot R, Kumari S. Burden of different beta-lactamase classes among clinical isolates of AmpC-producing Pseudomonas aeruginosa in burn patients: A prospective study. Indian J Crit Care Med. 2012;16(3):136-40.

23. Sreeshma P, Champa H, Sunil RP, Subbannayya K. Detection of extended spectrum $\beta$-lactamase, AmpC $\beta$-lactamase and metallo $\beta$-lactamase in clinical isolates of Pseudomonas aeruginosa. J Pharm Biomed Sci. 2013;33(33):1506-15.

24. Fazeli H, Sadighian H, Esfahani BN, Pourmand MR. Identification of class- 1 integron and various $\beta$-lactamase classes among clinical isolates of Pseudomonas aeruginosa at children's medical center hospital. J Med Bacteriol. 2012;1(2):25-36.

25. Laghawe A, Jaitly N, Thombare V. The Simultaneous Detection of the ESBL and the AmpC $\beta$-Lactamases in Gram Negative Bacilli. J Clin Diag Res. 2012;6(4suppl2):660-3.

26. Thomson KS. Controversies about extended-spectrum and AmpC beta-lactamases. Emerg Infect Dis. 2001;7(2):333-6.

27. Glupczynski Y, Bogaerts P, Deplano A, Berhin C, Huang TD, Van Eldere J, et al. Detection and characterization of class A extended-spectrum-beta-lactamase- producing Pseudomonas aeruginosa isolates in Belgian hospitals. J Antimicrob Chemother. 2010;65(5):866-71.

28. Imani Foloodi AA, Rostami Z, Shapouri R. Antimicrobial resistance and ESBL prevalence in Pseudomonas aeruginosa strains isolated from clinical specimen by phenotypic and genotypic methods. J Ardabil Univ Med Sci. 2010;10(37):189-98.

29. Jabalameli F, Mirsalehian A, Sotoudeh N, Jabalameli L, Aligholi M, Khoramian B, et al. Multiple-locus variable number of tandem repeats (VNTR) fingerprinting (MLVF) and antibacterial resistance profiles of extended spectrum beta lactamase (ESBL) producing Pseudomonas aeruginosa among burnt patients in Tehran. Burns. 2011;37(7):1202-7. 
30. Shacheraghi F, Shakibaie MR, Noveiri H. Molecular Identification of ESBL genes blaGES-1, blaVEB-1, blaCTX-M blaOXA-1, blaOXA-4, blaOXA-10 and blaPER-1 in Pseudomonas aeruginosa strains isolated from burn patients by PCR, RFLP and sequencing techniques. Int J Biol life Sci. 2010;3(6):138-42.

31. Shahcheraghi F, Nikbin VS, Feizabadi MM. Prevalence of ESBLs genes among multidrug-resistant isolates of Pseudomonas aeruginosa isolated from patients in Tehran. Microb Drug Resist. 2009;15(1):37-9.
32. Zaranza AV, Morais FC, do Carmo MS, de Mendonça Marques A Andrade-Monteiro C, Ferro TF, et al. Antimicrobial Susceptibility, Biofilm Production and Adhesion to HEp-2 Cells of Pseudomonas aeruginosa Strains Isolated from Clinical Samples.J Biomat Nanobiotechnol. 2013;4(1):98-106.

33. Bush K. Carbapenemases: Partners in crime.J Glob Antimicrob Res. 2013;1(1):7-16.

34. Livermore DM, Woodford N. Carbapenemases: a problem in waiting? Curr Opin Microbiol. 2000;3(5):489-95. 